Research Article

\title{
Hypersurfaces with Null Higher Order Anisotropic Mean Curvature
}

\author{
Hua Wang ${ }^{1}$ and Yijun $\mathrm{He}^{2}$ \\ ${ }^{1}$ School of Mathematical Sciences, Shanxi University, Taiyuan 030006, China \\ ${ }^{2}$ Research Institute of Mathematics and Applied Mathematics, Shanxi University, Taiyuan 030006, China \\ Correspondence should be addressed to Yijun He; sxheyijun@163.com
}

Received 18 April 2013; Accepted 11 June 2013

Academic Editor: Reza Saadati

Copyright (c) $2013 \mathrm{H}$. Wang and Y. He. This is an open access article distributed under the Creative Commons Attribution License, which permits unrestricted use, distribution, and reproduction in any medium, provided the original work is properly cited.

Given a positive function $F$ on $\mathbb{S}^{n}$ which satisfies a convexity condition, for $1 \leq r \leq n$, we define for hypersurfaces in $\mathbb{R}^{n+1}$ the $r$ th anisotropic mean curvature function $H_{r ; F}$, a generalization of the usual $r$ th mean curvature function. We call a hypersurface anisotropic minimal if $H_{F}=H_{1 ; F}=0$, and anisotropic $r$-minimal if $H_{r+1 ; F}=0$. Let $W$ be the set of points which are omitted by the hyperplanes tangent to $M$. We will prove that if an oriented hypersurface $M$ is anisotropic minimal, and the set $W$ is open and nonempty, then $x(M)$ is a part of a hyperplane of $\mathbb{R}^{n+1}$. We also prove that if an oriented hypersurface $M$ is anisotropic $r$-minimal and its $r$ th anisotropic mean curvature $H_{r ; F}$ is nonzero everywhere, and the set $W$ is open and nonempty, then $M$ has anisotropic relative nullity $n-r$.

\section{Introduction}

Let $F: \mathbb{S}^{n} \rightarrow \mathbb{R}^{+}$be a smooth function which satisfies the following convexity condition:

$$
\left(D^{2} F+F I\right)_{x}>0, \quad \forall x \in \mathbb{S}^{n}
$$

where $\mathbb{S}^{n}$ is the standard unit sphere in $\mathbb{R}^{n+1}, D^{2} F$ denotes the intrinsic Hessian of $F$ on $\mathbb{S}^{n}, I$ denotes the identity on $T_{x} \mathbb{S}^{n}$, and $>0$ means that the matrix is positive definite. We consider the map

$$
\begin{gathered}
\phi: \mathbb{S}^{n} \longrightarrow \mathbb{R}^{n+1}, \\
x \longrightarrow F(x) x+\left(\operatorname{grad}_{\mathbb{S}^{n}} F\right)_{x} ;
\end{gathered}
$$

its image $W_{F}=\phi\left(\mathbb{S}^{n}\right)$ is a smooth, convex hypersurface in $\mathbb{R}^{n+1}$ called the Wulff shape of $F$ (see [1-9]). When $F \equiv 1$, the Wulff shape $W_{F}$ is just $\mathbb{S}^{n}$.

Now let $x: M \rightarrow \mathbb{R}^{n+1}$ be a smooth immersion of an oriented hypersurface. Let $N: M \rightarrow \mathbb{S}^{n}$ denote its Gauss map. The map $v=\phi \circ N: M \rightarrow W_{F}$ is called the anisotropic Gauss map of $x$.
Let $S_{F}=-\mathrm{d} \nu . S_{F}$ is called the $F$-Weingarten operator, and the eigenvalues of $S_{F}$ are called anisotropic principal curvatures. Let $\sigma_{r}$ be the elementary symmetric functions of the anisotropic principal curvatures $\kappa_{1}, \kappa_{2}, \ldots, \kappa_{n}$ :

$$
\sigma_{r}=\sum_{i_{1}<\cdots<i_{r}} \kappa_{i_{1}} \cdots \kappa_{i_{r}} \quad(1 \leq r \leq n)
$$

We set $\sigma_{0}=1$. The $r$ th anisotropic mean curvature $H_{r ; F}$ is defined by $H_{r ; F}=\sigma_{r} / C_{n}^{r}$, also see Reilly [10]. $H_{F}:=H_{1 ; F}$ is called the anisotropic mean curvature. When $F \equiv 1, S_{F}$ is just the Weingarten operator of hypersurfaces, and $H_{r ; F}$ is just the $r$ th mean curvature $H_{r}$ of hypersurfaces which has been studied by many authors (see [11-14]). Thus, the $r$ th anisotropic mean curvature $H_{r ; F}$ generalizes the $r$ th mean curvature $H_{r}$ of hypersurfaces in the $(n+1)$-dimensional Euclidean space $\mathbb{R}^{n+1}$.

We say that $x: M \rightarrow \mathbb{R}^{n+1}$ is anisotropic $r$-minimal if $H_{r+1 ; F}=0$.

For $p \in M$, we define $v(p)=\operatorname{dim} \operatorname{ker}\left(S_{F}\right)$. We call $v=$ $\min _{p \in M} v(p)$ the anisotropic relative nullity; it generalized the usual relative nullity. 
For a smooth immersion $x: M \rightarrow \mathbb{Q}_{c}^{n+1}$ of a hypersurface into an $(n+1)$-dimensional space form with constant sectional curvature $c$, we denote by

$$
W=\mathbb{Q}_{c}^{n+1}-\bigcup_{p \in M}\left(\mathbb{Q}_{c}^{n}\right)_{p},
$$

where for every $p \in M,\left(\mathbb{Q}_{c}^{n}\right)_{p}$ is the totally geodesic hypersurface of $\mathbb{Q}_{c}^{n+1}$ tangent to $x(M)$ at $x(p)$. So, in the case of $c=$ $0, W$ is the set of points which are omitted by the hyperplanes tangent to $x(M)$.

We will study immersion with $W$ nonempty. In this direction, Hasanis and Koutroufiotis (see [15]) proved the following.

Theorem 1. Let $x: M \rightarrow \mathbb{Q}_{c}^{3}$ be a complete minimal immersion with $c \geq 0$. If $W$ is nonempty, then $x$ is totally geodesic.

Later, in [16], Alencar and Frensel extended the result above assuming an extra condition. They proved the following.

Theorem 2. Let $x: M \rightarrow \mathbb{Q}_{c}^{n+1}$ be an oriented, minimally immersed hypersurface. If $W$ is open and nonempty, then $x$ is totally geodesic.

In [17], Alencar and Batista studied hypersurfaces with null higher order mean curvature; they proved the following.

Theorem 3. Let $M$ be a complete and orientable Riemannian manifold and let $x: M \rightarrow \mathbb{Q}_{c}^{n+1}$ be an isometric immersion with $H_{r+1}=0$ and $H_{r} \neq 0$ everywhere, $r \geq 1$. If $W$ is open and nonempty, then the relative nullity $v=n-r$.

We note that, Alencar in [18] provides examples of nontotally geodesic minimal hypersurfaces in $\mathbb{R}^{2 n}, n \geq 4$, with nonempty $W$; in [17], Alencar and Batista provides examples of 1-minimal hypersurfaces with $H_{1} \neq 0$ everywhere in $\mathbb{R}^{2 n}, n \geq 5$, with nonempty $W$ but $v \neq n-1$. These examples show that it is necessary to add an extra hypothesis.

In this paper, we prove the anisotropic version of Theorems 2 and 3 for an immersion $x: M \rightarrow \mathbb{R}^{n+1}$. Explicitly, we prove the following two theorems.

Theorem 4. Let $x: M \rightarrow \mathbb{R}^{n+1}$ be an oriented, anisotropic minimally immersed hypersurface. If $W$ is open and nonempty, then $x(M)$ is a part of a hyperplane of $\mathbb{R}^{n+1}$.

Theorem 5. Let $x: M \rightarrow \mathbb{R}^{n+1}$ be an oriented immersed hypersurface with $H_{r+1 ; F}=0$ and $H_{r ; F} \neq 0$ everywhere, $r \geq 1$. If $W$ is open and nonempty, then the anisotropic relative nullity $v=n-r$.

\section{Preliminaries}

In this paper, we use the summation convention of Einstein and the following convention of index ranges unless otherwise stated:

$$
1 \leq i, j, \ldots \leq n ; \quad 1 \leq \alpha, \beta, \ldots \leq n+1 .
$$

We define $F^{*}: \mathbb{R}^{n+1} \rightarrow \mathbb{R}$ to be

$$
F^{*}(y)=\sup \left\{\frac{\langle y, z\rangle}{F(z)} \mid z \in \mathbb{R}^{n+1} \backslash\{0\}\right\} ;
$$

then $F^{*}$ is a Minkowski norm on $\mathbb{R}^{n+1}$. In fact, as proved in $[19], F^{*}: \mathbb{R}^{n+1} \backslash\{0\} \rightarrow \mathbb{R}$ is smooth and we have the following.

Proposition 6. (1) $F^{*}(y)>0$, for all $y \in \mathbb{R}^{n+1} \backslash\{0\}$;

(2) $F^{*}(t y)=t F^{*}(y)$, for all $y \in \mathbb{R}^{n+1}, t>0$;

(3) $F^{*}(y+z) \leq F^{*}(y)+F^{*}(z)$, for all $y, z \in \mathbb{R}^{n+1}$, and the equality holds if and only if $y=0$, or $z=0$ or $y=k z$ for some $k>0$.

(4) $W_{F}=\left\{y \in \mathbb{R}^{n+1} \mid F^{*}(y)=1\right\}$.

We define

$$
\begin{aligned}
& \bar{g}_{\alpha \beta}(y)=\frac{1}{2} \frac{\partial^{2}\left(F^{*}\right)^{2}}{\partial y^{\alpha} \partial y^{\beta}}(y), \\
& g_{y}(X, Y)=\bar{g}_{\alpha \beta}(y) X^{\alpha} Y^{\beta},
\end{aligned}
$$

where $y \in \mathbb{R}^{n+1} \backslash\{0\}$ and $X=\left(X^{1}, X^{2}, \ldots, X^{n+1}\right), Y=$ $\left(Y^{1}, Y^{2}, \ldots, Y^{n+1}\right) \in T_{y} \mathbb{R}^{n+1} \cong \mathbb{R}^{n+1}$.

From the Euler's theorem for homogeneous functions, we have

$$
\frac{\partial \bar{g}_{\alpha \beta}}{\partial y^{\gamma}}(z) z^{\beta}=\frac{1}{2} \frac{\partial^{3}\left(F^{*}\right)^{2}}{\partial y^{\alpha} \partial y^{\beta} \partial y^{\gamma}}(z) z^{\beta}=0,
$$

where $z=\left(z^{1}, z^{2}, \ldots, z^{n+1}\right) \in \mathbb{R}^{n+1} \backslash\{0\}$. Thus,

$$
\frac{\partial g_{z}(X, z)}{\partial y^{\gamma}}=\bar{g}_{\alpha \beta}(z) \frac{\partial X^{\alpha}}{\partial y^{\gamma}} z^{\beta}+\bar{g}_{\alpha \gamma}(z) X^{\alpha} \frac{\partial z^{\beta}}{\partial y^{\gamma}},
$$

where $z=\left(z^{1}, z^{2}, \ldots, z^{n+1}\right) \in T \mathbb{R}^{n+1}$ is nonzero everywhere and $X=\left(X^{1}, X^{2}, \ldots, X^{n+1}\right) \in T \mathbb{R}^{n+1}$.

As $F^{*}$ is a Minkowski norm on $\mathbb{R}^{n+1}$, the following lemma holds (see $[20,21])$.

Lemma 7. For any $y \in \mathbb{R}^{n+1} \backslash\{0\}$ and $u \in \mathbb{R}^{n+1}$ one has

$$
g_{y}(y, z) \leq F^{*}(y) F^{*}(z),
$$

and the equality holds if and only if there exists $t \geq 0$ such that $z=t y$.

Let $x: M \rightarrow \mathbb{R}^{n+1}$ be an oriented hypersurface in the Euclidean space $\mathbb{R}^{n+1}$. Let $v: M \rightarrow W_{F}$ denote its anisotropic Gauss map. Then for any $p \in M, v(p)$ is perpendicular to $x_{*}\left(T_{p} M\right)$ with respect to the inner product $g_{v(p)}$ and $F^{*}(\nu(p))=1$. Thus, we call $\nu(p)$ an anisotropic unit normal vector of $T_{p} M$.

\section{A Connection on Hypersurfaces of Minkowski Space}

Let $x: M \rightarrow \mathbb{R}^{n+1}$ be an oriented hypersurface in the Euclidean space $\mathbb{R}^{n+1}$ and denote $v: M \rightarrow W_{F}$ its anisotropic Gauss map. 
Let $\bar{\nabla}$ be the standard connection on the $(n+1)$-dimensional Euclidean space $\mathbb{R}^{n+1}$. For vector fields $X, Y$ on $M$, we decompose $\bar{\nabla}_{X} Y$ as the tangent part $\nabla_{X} Y$ and the anisotropic normal part II $(X, Y) v$ with respect to the inner product $g_{v}$. That is,

$$
\bar{\nabla}_{X} Y=\nabla_{X} Y+\operatorname{II}(X, Y) \nu,
$$

where $g_{\nu}\left(\nabla_{X} Y, \nu\right)=0$.

We also have the Weingarten formula:

$$
\begin{gathered}
\bar{\nabla}_{X} v=-S_{F} X, \\
g_{\nu}\left(S_{F} X, Y\right)=\operatorname{II}(X, Y),
\end{gathered}
$$

where we have used (9).

It is easy to verify that $\nabla$ is a torsion free connection on $M$ and II is a symmetric second order covariant tensor field on $M$. We call II the anisotropic second fundamental form.

Let $\left\{e_{i}\right\}_{i=1}^{n}$ be a local frame of $M$ and $\left\{\omega^{i}\right\}_{i=1}^{n}$ its dual frame. Let $g_{i j}=g_{v}\left(e_{i}, e_{j}\right), \nabla e_{i}=\omega_{i}^{j} \otimes e_{j}, \operatorname{II}\left(e_{i}, e_{j}\right)=h_{i j}, h_{i}^{j}=g^{j k} h_{k i}$, where $\left(g^{i j}\right)$ is the inverse matrix of $\left(g_{i j}\right)$. Then we have

$$
\begin{gathered}
d x=\omega^{i} e_{i}, \\
d e_{i}=\omega_{i}^{j} e_{j}+h_{i j} \omega^{j} \nu, \\
d \nu=-h_{i}^{j} \omega^{i} e_{j} .
\end{gathered}
$$

Differentiating (13) and using (14), we get

$$
\begin{gathered}
d \omega^{i}=\omega^{j} \wedge \omega_{j}^{i}, \\
h_{i j}=h_{j i} .
\end{gathered}
$$

Differentiating (14) and using (14)-(15), we get

$$
\begin{gathered}
h_{i j k}=h_{i k j}, \\
d \omega_{i}^{j}-\omega_{i}^{k} \wedge \omega_{k}^{j}=-\frac{1}{2} R_{i k l}^{j} \omega^{k} \wedge \omega^{l},
\end{gathered}
$$

where

$$
d h_{i j}-h_{i k} \omega_{j}^{k}-h_{k j} \omega_{i}^{k}=h_{i j k} \omega^{k},
$$

and $R_{i k l}^{j}=-R_{i l k}^{j}=h_{i k} h_{l}^{j}-h_{i l} h_{k}^{j}$.

Differentiating (15) and using (14), we get

$$
h_{i k}^{j}=h_{k i}^{j} \text {, }
$$

where

$$
d h_{i}^{j}+h_{i}^{k} \omega_{k}^{j}-h_{k}^{j} \omega_{i}^{k}=h_{i k}^{j} \omega^{k} .
$$

Note $\left(h_{i}^{j}\right)$ is the matrix of the F-Weingarten operator $S_{F}=-d v$, its eigenvalues are called the anisotropic principal curvatures, and we denote them by $\kappa_{1}, \ldots, \kappa_{n}$.

We have $n$ invariants, the elementary symmetric function $\sigma_{r}$ of the anisotropic principal curvatures:

$$
\sigma_{r}=\sum_{i_{1}<\cdots i_{r}} \kappa_{i_{1}} \cdots \kappa_{i_{n}} \quad(1 \leq r \leq n) .
$$

For convenience, we set $\sigma_{0}=1$. The $r$ th anisotropic mean curvature $H_{r ; F}$ is defined by

$$
H_{r ; F}=\frac{\sigma_{r}}{C_{n}^{r}}, \quad C_{n}^{r}=\frac{n !}{r !(n-r) !} .
$$

Using the characteristic polynomial of $S_{F}, \sigma_{r}$ is defined by

$$
\operatorname{det}\left(t I-S_{F}\right)=\sum_{r=0}^{n}(-1)^{r} \sigma_{r} t^{n-r} .
$$

So, we have

$$
\sigma_{r}=\frac{1}{r !} \sum_{i_{1}, \ldots, i_{r} ; j_{1}, \ldots, j_{r}} \delta_{i_{1} \cdots i_{r}}^{j_{1} \cdots j_{r}} h_{j_{1}}^{i_{1}} \cdots h_{j_{r}}^{i_{r}}
$$

where $\delta_{i_{1} \cdots i_{r}}^{j_{1} \cdots j_{r}}$ is the usual generalized Kronecker symbol; that is, $\delta_{i_{1} \cdots i_{r}}^{j_{1} \cdots j_{r}}$ equals +1 (resp., -1 ) if $i_{1} \cdots i_{r}$ are distinct and $\left(j_{1} \cdots j_{r}\right)$ is an even (resp., odd) permutation of $\left(i_{1} \cdots i_{r}\right)$ and in other cases it equals zero.

Definition 8. Let $f: M \rightarrow \mathbb{R}$ be a smooth function. One defines the gradient (with respect to the induced metric $g_{v}$ on $M) \operatorname{grad} f$ of the function $f$ by

$$
g_{v}(\operatorname{grad} f, X)=X(f),
$$

where $X$ is any smooth vector field on $M$.

Define $f_{i}$ by $d f=f_{i} \omega^{i}$; then

$$
\operatorname{grad} f=g^{i j} f_{j} e_{i} .
$$

We define

$$
d V=\left|e_{1}, \ldots, e_{n}, v\right| \omega^{1} \wedge \cdots \wedge \omega^{n},
$$

where $\left|e_{1}, \ldots, e_{n}, \nu\right|$ is the determinant of the matrix $\left(e_{1}\right.$, $\left.\ldots, e_{n}, v\right)$. Then $d V$ is a volume element on $M$.

Definition 9. Let $X$ be a smooth vector field on $M$. One defines the divergence (with respect to the volume element $d V) \operatorname{div} X$ by $d\{i(X) d V\}=(\operatorname{div} X) d V$, where

$$
\begin{aligned}
&(i(X) d V)\left(Y_{1}, \ldots, Y_{n-1}\right) \equiv d V\left(X, Y_{1}, \ldots, Y_{n-1}\right), \\
& \forall Y_{1}, \ldots, Y_{n-1} \in \mathscr{X}(M) .
\end{aligned}
$$

Lemma 10. Let $X=X^{i} e_{i}$; then $\operatorname{div} X=X_{i}^{i}$, where

$$
d X^{i}+X^{j} \omega_{j}^{i}=X_{j}^{i} \omega^{j} .
$$

Proof. By (14), (15), we get

$$
d\left|e_{1}, \ldots, e_{n}, v\right|=\omega_{i}^{i}\left|e_{1}, \ldots, e_{n}, v\right| .
$$

From the definition of $i(X)$, we have

$$
\begin{gathered}
i(X) d V=\sum_{i}(-1)^{i+1} X^{i}\left|e_{1}, \ldots, e_{n}, \nu\right| \omega^{1} \\
\wedge \cdots \wedge \widehat{\omega}^{i} \wedge \cdots \wedge \omega^{n} .
\end{gathered}
$$


So,

$$
\begin{aligned}
d\{i(X) d V\}=\sum_{i}(-1)^{i+1}\left(d X^{i}\right) \wedge\left|e_{1}, \ldots, e_{n}, \nu\right| \omega^{1} & \wedge \wedge \widehat{\omega}^{i} \wedge \cdots \wedge \omega^{n} \\
& +\sum_{i}(-1)^{i+1} X^{i}\left(d\left|e_{1}, \ldots, e_{n}, \nu\right|\right) \\
& \wedge \omega^{1} \wedge \cdots \wedge \widehat{\omega}^{i} \wedge \cdots \wedge \omega^{n} \\
& +\sum_{j<i}(-1)^{i+j} X^{i}\left|e_{1}, \ldots, e_{n}, \nu\right| d \omega^{j} \wedge \omega^{1} \\
& \wedge \cdots \wedge \widehat{\omega}^{j} \wedge \cdots \wedge \widehat{\omega}^{i} \wedge \cdots \wedge \omega^{n} \\
& +\sum_{j>i}(-1)^{i+j+1} X^{i}\left|e_{1}, \ldots, e_{n}, \nu\right| d \omega^{j} \\
& \wedge \omega^{1} \wedge \cdots \wedge \widehat{\omega}^{i} \wedge \cdots \wedge \widehat{\omega}^{j} \wedge \cdots \wedge \omega^{n} \\
= & X_{i}^{i} d V .
\end{aligned}
$$

\section{4. $L_{r ; F}$ Operator for Hypersurfaces}

We introduce the Newton transformation defined by

$$
P_{r}=\sigma_{r} I-\sigma_{r-1} S_{F}+\cdots+(-1)^{r} S_{F}^{r}, \quad r=0, \ldots, n
$$

then

$$
P_{0}=I, \quad P_{n}=0, \quad P_{r}=\sigma_{r} I-P_{r-1} S_{F} .
$$

Lemma 11. The matrix of $P_{r}$ is given by:

$$
\left(P_{r}\right)_{i}^{j}=\frac{1}{r !} \delta_{i_{1} \cdots i_{r} i}^{j_{1} \cdots j_{r} j} h_{j_{1}}^{i_{1}} \cdots h_{j_{r}}^{i_{r}}
$$

Proof. We prove Lemma 11 inductively. For $r=0$, it is easy to check that (35) is true.

We can check directly

$$
\delta_{i_{1} \cdots i_{q}}^{j_{1} \cdots j_{q}}=\left|\begin{array}{ccccc}
\delta_{i_{1}}^{j_{1}} & \delta_{i_{1}}^{j_{2}} & \cdots & \delta_{i_{1}}^{j_{q_{q}-1}} & \delta_{i_{1}}^{j_{q}} \\
\delta_{i_{2}}^{j_{1}} & \delta_{i_{2}}^{j_{2}} & \cdots & \delta_{i_{2}}^{j_{q-1}} & \delta_{i_{2}}^{j_{q}} \\
\vdots & \vdots & \ddots & \vdots & \vdots \\
\delta_{i_{q-1}}^{j_{1}} & \delta_{i_{q-1}}^{j_{2}} & \cdots & \delta_{i_{q-1}}^{j_{q-1}} & \delta_{i_{q-1}}^{j_{q}} \\
\delta_{i_{q}}^{j_{1}} & \delta_{i_{q}}^{j_{2}} & \cdots & \delta_{i_{q}}^{j_{q-1}} & \delta_{i_{q}}^{j_{q}}
\end{array}\right| .
$$

Assume that (35) is true for $r=k$, we only need to show that it is also true for $r=k+1$. For $r=k+1$, using (24) and (36), we have

RHS of (35)

$$
\begin{aligned}
& =\frac{1}{(k+1) !} \sum_{i_{1}, \ldots, i_{k+1} ; j_{1}, \ldots, j_{k+1}} \delta_{i_{1} \cdots i_{k+1} i}^{j_{1} \cdots j_{k+1} j} h_{i_{1}}^{j_{1}} \cdots h_{i_{k+1}}^{j_{k+1}} \\
& =\frac{1}{(k+1) !} \sum\left|\begin{array}{ccccc}
\delta_{i_{1}}^{j_{1}} & \delta_{i_{1}}^{j_{2}} & \cdots & \delta_{i_{1}}^{j_{k+1}} & \delta_{i_{1}}^{j} \\
\delta_{i_{2}}^{j_{1}} & \delta_{i_{2}}^{j_{2}} & \cdots & \delta_{i_{2}}^{j_{k+1}} & \delta_{i_{2}}^{j} \\
\vdots & \vdots & \ddots & \vdots & \vdots \\
\delta_{i_{k+1}}^{j_{1}} & \delta_{i_{k+1}}^{j_{2}} & \cdots & \delta_{i_{k+1}}^{j_{k+1}} & \delta_{i_{k+1}}^{j} \\
\delta_{i}^{j_{1}} & \delta_{i}^{j_{2}} & \cdots & \delta_{i}^{j_{k+1}} & \delta_{i}^{j}
\end{array}\right| h_{i_{1}}^{j_{1}} \cdots h_{i_{k+1}}^{j_{k+1}} \\
& =\frac{1}{(k+1) !} \sum\left(\delta_{i}^{j} \delta_{i_{1} \cdots i_{k+1}}^{j_{1} \cdots j_{k+1}}-\delta_{i}^{j_{k+1}} \delta_{i_{1} \cdots i_{k} i_{k+1}}^{j_{1} \cdots j_{k} j}+\cdots\right) h_{i_{1}}^{j_{1}} \cdots h_{i_{k+1}}^{j_{k+1}} \\
& =\sigma_{k+1} \delta_{i}^{j}-\frac{1}{(k+1) !} \sum \delta_{i}^{j_{k+1}} \delta_{i_{1} \cdots i_{k} i_{k+1}}^{j_{1} \cdots j_{k} j} h_{i_{1}}^{j_{1}} \cdots h_{i_{k+1}}^{j_{k+1}}+\cdots \\
& =\sigma_{k+1} \delta_{i}^{j}-\sum\left(P_{k}\right)_{i}^{i_{k+1}} h_{i_{k+1}^{j}}^{j} \\
& =\left(P_{k+1}\right)_{i}^{j} \text {. }
\end{aligned}
$$

Lemma 12. For each $r$, one has
(a) $\left(P_{r}\right)_{i j}{ }^{j}=0$;
(b) $\operatorname{Trace}\left(P_{r} S_{F}\right)=(r+1) \sigma_{r+1}$;
(c) $\operatorname{Trace}\left(P_{r}\right)=(n-r) \sigma_{r}$;
(d) $\operatorname{Trace}\left(P_{r} S_{F}^{2}\right)=\sigma_{1} \sigma_{r+1}-(r+2) \sigma_{r+2}$.

Proof. (a) Noting $\left(j, j_{r}\right)$ is skew symmetric in $\delta_{i_{1} \cdots i_{r} i}^{j_{1} \cdots j_{j} j}$ and $\left(j, j_{r}\right)$ is symmetric in $h_{j_{1}}^{i_{1}} \cdots h_{j_{r} j}{ }_{r_{r}}$ (from (19), we have

$$
\sum_{j}\left(P_{r}\right)_{i j}{ }^{j}=\frac{1}{(r-1) !} \sum_{i_{1}, \ldots, i_{r} ; j_{1}, \ldots, j_{r} ; j} \delta_{i_{1} \cdots i_{r} i}^{j_{1} \cdots j_{r} j} h_{j_{1}}^{i_{1}} \cdots h_{j_{r} j}^{{ }^{i_{r}}}=0 .
$$

(b) Using (35) and (24), we have

$$
\begin{aligned}
\operatorname{Trace}\left(P_{r} S_{F}\right) & =\sum_{i j}\left(P_{r}\right)_{i}^{j} h_{j}^{i} \\
& =\frac{1}{r !} \sum_{i_{1}, \ldots, i_{r} ; j_{1}, \ldots, j_{r} ; i, j} \delta_{i_{1} \cdots i_{r} i}^{j_{1} \cdots j_{r} j} h_{j_{1}}^{i_{1}} \cdots h_{j_{r}}^{i_{r}} h_{j}^{i} \\
& =(r+1) \sigma_{r+1} .
\end{aligned}
$$

(c) Using (b) and the definition of $P_{r}$, we have

$\operatorname{Trace}\left(P_{r}\right)=\operatorname{tr}\left(\sigma_{r} I\right)-\operatorname{tr}\left(P_{r-1} S_{F}\right)=n \sigma_{r}-r \sigma_{r}=(n-r) \sigma_{r}$.

(d) Using (b) and the definition of $P_{r+1}$, we have

$$
\begin{aligned}
\operatorname{Trace}\left(P_{r} S_{F}^{2}\right) & =\operatorname{Trace}\left(\sigma_{r+1} S_{F}\right)-\operatorname{Trace}\left(P_{r+1} S_{F}\right) \\
& =\sigma_{1} \sigma_{r+1}-(r+2) \sigma_{r+2} .
\end{aligned}
$$


Remark 13. When $F=1$, Lemma 12 was a well-known result (e.g., see Barbosa and Colares [22], or Reilly [23]).

Lemma 14. One has

$$
\left(\sigma_{r}\right)_{k}=\sum_{i, j}\left(P_{r-1}\right)_{i}^{j} h_{j_{k}}^{i} .
$$

Proof. From the definition of $\sigma_{r}$, we have the following calculation:

$$
\begin{aligned}
\left(\sigma_{r}\right)_{k} & =\frac{1}{r !} \sum_{i_{1}, \ldots, i_{r} ; j_{1}, \ldots, j_{r}} \delta_{i_{1} \cdots i_{r}}^{j_{1} \cdots j_{r}}\left(h_{i_{1}}^{j_{1}} \cdots h_{i_{r}}^{j_{r}}\right)_{k} \\
& =\frac{1}{(r-1) !} \sum_{i_{1}, \ldots, i_{r} ; j_{1}, \ldots, j_{r}} \delta_{i_{1} \cdots i_{r}}^{j_{1} \cdots j_{r}} h_{i_{1}}^{j_{1}} \cdots h_{i_{r} k}^{j_{r}} \\
& =\sum_{i_{r}, j_{r}}\left(P_{r-1}\right)_{i_{r}}^{j_{r}} h_{i_{r} k}^{j_{r}}=\sum_{i, j}\left(P_{r-1}\right)_{i}^{j_{i}} h_{i k}^{j} .
\end{aligned}
$$

We define an operator $L_{r ; F}: C^{\infty}(M) \rightarrow C^{\infty}(M)$ by

$$
L_{r ; F}(f)=\operatorname{div}\left(P_{r} \nabla f\right) .
$$

In the sequel, we will need the following lemma. Item (a) is essentially the content of Lemma 1.1 and Equation (1.3) in [24], while item (b) is quoted as Proposition 1.5 in [25].

Lemma 15. Let $x: M \rightarrow \mathbb{R}^{n+1}$ be an oriented hypersurface, and $0 \leq r \leq n-1, p \in M$.

(a) If $\sigma_{r+1}(p)=0$, then $P_{r}$ is semidefinite at $p$;

(b) if $\sigma_{r+1}(p)=0$ and $\sigma_{r+2}(p) \neq 0$, then $P_{r}$ is definite at $p$.

Another important result is as follows (see [26]).

Lemma 16. Let $x: M \rightarrow \mathbb{R}^{n+1}$ be an oriented hypersurface, and $p \in M$.

(a) For $1 \leq r \leq n$, one has $H_{r ; F}^{2} \geq H_{r-1 ; F} H_{r+1 ; F}$. Moreover, if equality happens for $r=1$ or for some $1<r<n$, with $H_{r+1 ; F} \neq 0$ in this case, then $p$ is an anisotropic umbilical point (i.e. $\kappa_{1}(p)=\kappa_{2}(p)=\cdots=\kappa_{n}(p)$ );

(b) if, for some $1 \leq r<n$, one has $H_{r ; F}=H_{r+1 ; F}=0$, then $H_{j ; F}=0$ for all $r \leq j \leq n$. In particular, at most $r-1$ of the anisotropic principal curvatures are different from zero.

The result below is standard, so we omit the proof.

Lemma 17. Let $x: M \rightarrow \mathbb{R}^{n+1}$ be an oriented hypersurface. The operator $L_{r ; F}$ associated to the immersion $x$ is elliptic if and only if $P_{r}$ is positive definite.

Definition 18. Let $f: M \rightarrow \mathbb{R}$ be a smooth function. The Laplacian $\Delta f$ is defined by $\Delta f:=L_{0 ; F} f=\operatorname{div}(\operatorname{grad} f)$.

It is easy to see that $\Delta$ is an elliptic differential operator.
Definition 19. Let $x: M \rightarrow \mathbb{R}^{n+1}$ be an immersed hypersurface and $v$ its anisotropic unit normal vector field. The function $u:=g_{v}(x, v)$ is called the support function of the immersion $x$.

Next, we compute $L_{r ; F} u$ for the support function $u=$ $g_{v}(x, v)$.

Differentiating the decomposition

$$
x=g^{i j} g_{v}\left(x, e_{i}\right) e_{j}+u v,
$$

we obtain

$$
\begin{aligned}
d x= & \left\{d\left(g^{i j} g_{\nu}\left(x, e_{j}\right)\right)\right\} e_{i}+g^{i j} g_{\nu}\left(x, e_{j}\right) d e_{i} \\
& +(d u) \nu+u d v .
\end{aligned}
$$

So, from (13), (14), and (15) we have

$$
\begin{aligned}
\omega^{i} e_{i}= & \left\{d\left(g^{i j} g_{v}\left(x, e_{j}\right)\right)+g^{k j} g_{v}\left(x, e_{j}\right) \omega_{k}^{i}-u h_{j}^{i} \omega^{j}\right\} e_{i} \\
& +\left(d u+g^{j k} g_{v}\left(x, e_{j}\right) h_{i k} \omega^{i}\right) \nu .
\end{aligned}
$$

Thus, we get

$$
\begin{gathered}
d u=-g^{j k} g_{v}\left(x, e_{j}\right) h_{i k} \omega^{i}, \\
d\left(g^{i j} g_{v}\left(x, e_{j}\right)\right)+g^{k j} g_{v}\left(x, e_{j}\right) \omega_{k}^{i}-u h_{j}^{i} \omega^{j}=\omega^{i} .
\end{gathered}
$$

Denote $u^{i},\left(g^{i j} g_{v}\left(x, e_{j}\right)\right)_{k}, u_{j}^{i}$ by

$$
\begin{gathered}
\operatorname{grad} u=u^{i} e_{i}, \\
\left(g^{i j} g_{v}\left(x, e_{j}\right)\right)_{k} \omega^{k}=d\left(g^{i j} g_{v}\left(x, e_{j}\right)\right)+\left(g^{k j} g_{v}\left(x, e_{j}\right)\right) \omega_{k}^{i}, \\
u_{j}^{i} \omega^{j}=d u^{i}+u^{j} \omega_{j}^{i},
\end{gathered}
$$

respectively. Then we have (using (19) the following

$$
\begin{aligned}
& u^{i}=-g^{i l} h_{k l} g^{j k} g_{v}\left(x, e_{j}\right)=-h_{k}^{i} g^{k l} g_{v}\left(x, e_{l}\right), \\
&\left(g^{i k} g_{v}\left(x, e_{k}\right)\right)_{j}=\delta_{j}^{i}+h_{j}^{i} g_{v}(x, v), \\
& u_{j}^{i}=-h_{k j}^{i} g^{k l} g_{v}\left(x, e_{l}\right)-h_{k}^{i}\left(g^{k l} g_{v}\left(x, e_{l}\right)\right)_{j} \\
&=-h_{j_{k}}^{i} g^{k l} g_{v}\left(x, e_{l}\right)-h_{j}^{i}-h_{k}^{i} h_{j}^{k} u .
\end{aligned}
$$

By using Lemmas 12 and 14, we get

$$
\begin{aligned}
L_{r ; F} u= & \left(P_{r}\right)_{i}^{j} u_{j}^{i} \\
= & -\left(P_{r}\right)_{i}^{j} h_{j_{k}}^{i} g^{k l} g_{v}\left(x, e_{l}\right) \\
& -\left(P_{r}\right)_{i}^{j} h_{j}^{i}-\left(P_{r}\right)_{i}^{j} h_{k}^{i} h_{j}^{k} u \\
= & -\left(\sigma_{r+1}\right)_{k} g^{k l} g_{v}\left(x, e_{l}\right)-\left(P_{r}\right)_{i}^{j} h_{j}^{i}-\left(P_{r}\right)_{i}^{j} h_{k}^{i} h_{j}^{k} u \\
= & -g_{v}\left(\nabla \sigma_{r+1}, x\right)-(r+1) \sigma_{r+1} \\
& -\left(\sigma_{1} \sigma_{r+1}-(r+2) \sigma_{r+2}\right) u .
\end{aligned}
$$

Thus, we proved the following lemma. 
Lemma 20. For $0 \leq r \leq n-1$, one has the following

$$
\begin{aligned}
L_{r ; F} u= & -g_{v}\left(\nabla \sigma_{r+1}, x\right)-(r+1) \sigma_{r+1} \\
& -\left(\sigma_{1} \sigma_{r+1}-(r+2) \sigma_{r+2}\right) u .
\end{aligned}
$$

Remark 21. Recall $\sigma_{1}=n H_{F}$ and $|\mathrm{II}|^{2}=\sigma_{1}^{2}-2 \sigma_{2}$; let $r=0$ in (52); we get

$$
\Delta u=-n\left(H_{F}+g_{v}\left(\operatorname{grad} H_{F}, x\right)\right)-|\mathrm{II}|^{2} u .
$$

\section{Proof of Theorems 4 and 5}

We fix a point $o \in W$ as the origin of $\mathbb{R}^{n+1}$. Without loss of generality, we assume, for each $p \in M, v(p)$ is the anisotropic unit normal vector of $x(M)$ at $x(p)$ such that $\langle x(p), v(p)\rangle_{\nu(p)}>0$ (otherwise we consider the function $-u$ instead). This gives an orientation to $M$; indeed, the component of the position vector $x$ perpendicular (with respect to the inner product $g_{v}$ ) to $M$ defines a never zero, anisotropic normal, vector field on $M$, such that the support function $u=\langle x(p), v(p)\rangle_{\nu(p)}$ is positive on $M$.

5.1. Proof of Theorem 4. Since $x$ is anisotropic minimal, from (53) we get

$$
\Delta u=-|\mathrm{II}|^{2} u \leq 0, \quad \text { on } M .
$$

Let $u_{*}=\inf _{M} u$. We claim that $u_{*}$ is attained at some point $x_{0} \in M$. Consider a sequence $\left\{x_{k}\right\} \subset M$ such that $u\left(x_{k}\right) \rightarrow$ $u_{*}$ as $k \rightarrow+\infty$. To each $x_{k}$ we associate $y_{k}=u\left(x_{k}\right) \nu\left(x_{k}\right)$; then $y_{k} \in T_{x_{k}} M$. Since $\left\|y_{k}\right\|_{\mathbb{R}^{n+1}}=u\left(x_{k}\right)\left\|v\left(x_{k}\right)\right\|_{\mathbb{R}^{n+1}}$ is bounded, there exists a subsequence, which again we call $\left\{y_{k}\right\}$, such that $y_{k} \rightarrow y_{0}$ for some $y_{0} \in \mathbb{R}^{n+1}$. Since $\bigcup_{p \in M} T_{p} M$ is closed and $\left\{y_{k}\right\} \subset_{p \in M} T_{p} M$ we deduce that $y_{0} \in T_{x_{0}} M$ for some $x_{0} \in M$. Thus, by the continuity of $F^{*}$ and Lemma 7 ,

$$
\begin{aligned}
u_{*} & =\lim _{k \rightarrow+\infty} u\left(x_{k}\right)=\lim _{k \rightarrow+\infty} F^{*}\left(y_{k}\right) \\
& =F^{*}\left(y_{0}\right) \geq g_{v\left(x_{0}\right)}\left(y_{0}, v\left(x_{0}\right)\right)=u\left(x_{0}\right),
\end{aligned}
$$

so $u^{*}=u\left(x_{0}\right)$ as needed. Now, from the usual maximum principle $u$ is constant, $u=u_{*}=u\left(x_{0}\right)>0$. From (54) we then have II $\equiv 0$ and $x$ is totally geodesic.

\subsection{Proof of Theorem 5. Since $H_{r+1 ; F}=0$, from Lemma 20 we} get

$$
L_{r ; F} u=(r+2) \sigma_{r+2} u .
$$

Using Lemma 15(a) we have that $P_{r}$ is semidefinite. Since $H_{r ; F}$ does not vanish, we have that $H_{r ; F}$ is positive or negative, because $c(r) H_{r ; F}=\operatorname{Trace}\left(P_{r}\right)$, where $c(r)=(n-r) C_{n}^{r}$. Now we use Lemma 16 and obtain the following:

$$
0=H_{r+1 ; F}^{2} \geq H_{r ; F} H_{r+2 ; F} .
$$

Using the information above, we claim that $H_{r+2 ; F} \equiv 0$.

Case (i) $\left(H_{r ; F}>0\right)$. In this case, $P_{r}$ is positive definite, and $L_{r ; F}$ is elliptic by Lemma 17. Using (57) we conclude that $H_{r+2 ; F} \leq$ 0 . Whereas from (56) we have

$$
L_{r ; F} u \leq 0 .
$$

Following exactly the proof as in Theorem 4, we conclude that $u$ is constant, $u=u_{*}=u\left(x_{0}\right)>0$. From (56) we then have $H_{r+2 ; F} \equiv 0$.

Case (ii) $\left(H_{r ; F}<0\right)$. In this case, $P_{r}$ is negative definite, and $-L_{r ; F}$ is elliptic by Lemma 17. Using (57) we conclude that $H_{r+2 ; F} \geq 0$. Whereas from (56) we have

$$
-L_{r ; F} u \leq 0 \text {. }
$$

Now, following exactly the proof as in Theorem 4, we conclude that $u$ is constant, $u=u_{*}=u\left(x_{0}\right)>0$. From (56) we then have $H_{r+2 ; F} \equiv 0$.

Thus we conclude that $H_{r+2 ; F} \equiv 0$. Now, we use Lemma 16(b) to conclude that $H_{j ; F}=0$ for $j \geq r+1$ and so that $v \geq$ $n-r$. Since $H_{r ; F}$ does not change sign we have that $v=n-r$.

\section{References}

[1] J. E. Brothers and F. Morgan, "The isoperimetric theorem for general integrands," The Michigan Mathematical Journal, vol. 41, no. 3, pp. 419-431, 1994.

[2] U. Clarenz, "The Wulff shape minimizes an anisotropic Willmore functional," Interfaces and Free Boundaries. Mathematical Modelling, Analysis and Computation, vol. 6, no. 3, pp. 351-359, 2004.

[3] M. Koiso and B. Palmer, "Geometry and stability of surfaces with constant anisotropic mean curvature," Indiana University Mathematics Journal, vol. 54, no. 6, pp. 1817-1852, 2005.

[4] M. Koiso and B. Palmer, "Stability of anisotropic capillary surfaces between two parallel planes," Calculus of Variations and Partial Differential Equations, vol. 25, no. 3, pp. 275-298, 2006.

[5] M. Koiso and B. Palmer, "Anisotropic capillary surfaces with wetting energy," Calculus of Variations and Partial Differential Equations, vol. 29, no. 3, pp. 295-345, 2007.

[6] M. Koiso and B. Palmer, "Uniqueness theorems for stable anisotropic capillary surfaces," SIAM Journal on Mathematical Analysis, vol. 39, no. 3, pp. 721-741, 2007.

[7] F. Morgan, "Planar Wulff shape is unique equilibrium," Proceedings of the American Mathematical Society, vol. 133, no. 3, pp. 809-813, 2005.

[8] B. Palmer, "Stability of the Wulff shape," Proceedings of the American Mathematical Society, vol. 126, no. 12, pp. 3661-3667, 1998.

[9] J. E. Taylor, "Crystalline variational problems," Bulletin of the American Mathematical Society, vol. 84, no. 4, pp. 568-588, 1978.

[10] R. C. Reilly, "The relative differential geometry of nonparametric hypersurfaces," Duke Mathematical Journal, vol. 43, no. 4, pp. 705-721, 1976.

[11] L. Cao and H. Li, " $r$-minimal submanifolds in space forms," Annals of Global Analysis and Geometry, vol. 32, no. 4, pp. 311341, 2007.

[12] H. Li, "Hypersurfaces with constant scalar curvature in space forms," Mathematische Annalen, vol. 305, no. 4, pp. 665-672, 1996.

[13] S. Montiel and A. Ros, "Compact hypersurfaces: the Alexandrov theorem for higher order mean curvatures," in Differential geometry, B. Lawson and K. Tenenblat, Eds., vol. 52, pp. 279296, Longman, Harlow, UK, 1991. 
[14] A. Ros, "Compact hypersurfaces with constant higher order mean curvatures," Revista Matemática Iberoamericana, vol. 3, no. 3-4, pp. 447-453, 1987.

[15] T. Hasanis and D. Koutroufiotis, "A property of complete minimal surfaces," Transactions of the American Mathematical Society, vol. 281, no. 2, pp. 833-843, 1984.

[16] H. Alencar and K. Frensel, "Hypersurfaces whose tangent geodesics omit a nonempty set," in Pitman Monographs, vol. 52, pp. 1-13, Surveys in Pure and Applied Mathematics, 1991.

[17] H. Alencar and M. Batista, "Hypersurfaces with null higher order mean curvature," Bulletin of the Brazilian Mathematical Society, vol. 41, no. 4, pp. 481-493, 2010.

[18] H. Alencar, Hipersuperfícies Mnimas de $\mathbb{R}^{2 m}$ Invariantes por $\mathrm{SO}(\mathrm{m}), \mathrm{SO}(\mathrm{m})$ [Doctor thesis], IMPA-Brazil, 1988.

[19] Y. He, H. Li, H. Ma, and J. Ge, "Compact embedded hypersurfaces with constant higher order anisotropic mean curvatures," Indiana University Mathematics Journal, vol. 58, no. 2, pp. 853868, 2009.

[20] D. Bao, S.-S. Chern, and Z. Shen, An Introduction to RiemannFinsler Geometry, Springer, New York, NY, USA, 2000.

[21] Z. Shen, Lectures on Finsler Geometry, World Scientific, Singapore, 2001.

[22] J. L. M. Barbosa and A. G. Colares, "Stability of hypersurfaces with constant $r$-mean curvature," Annals of Global Analysis and Geometry, vol. 15, no. 3, pp. 277-297, 1997.

[23] R. C. Reilly, "Variational properties of functions of the mean curvatures for hypersurfaces in space forms," Journal of Differential Geometry, vol. 8, pp. 465-477, 1973.

[24] J. Hounie and M. L. Leite, "The maximum principle for hypersurfaces with vanishing curvature functions," Journal of Differential Geometry, vol. 41, no. 2, pp. 247-258, 1995.

[25] J. Hounie and M. L. Leite, “Two-ended hypersurfaces with zero scalar curvature," Indiana University Mathematics Journal, vol. 48 , no. 3, pp. 867-882, 1999.

[26] A. Caminha, "On spacelike hypersurfaces of constant sectional curvature lorentz manifolds," Journal of Geometry and Physics, vol. 56, no. 7, pp. 1144-1174, 2006. 


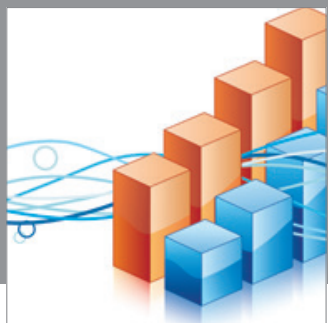

Advances in

Operations Research

mansans

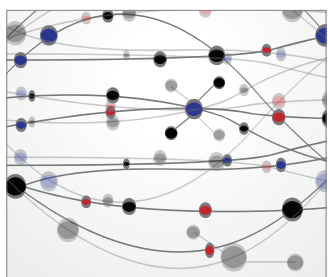

The Scientific World Journal
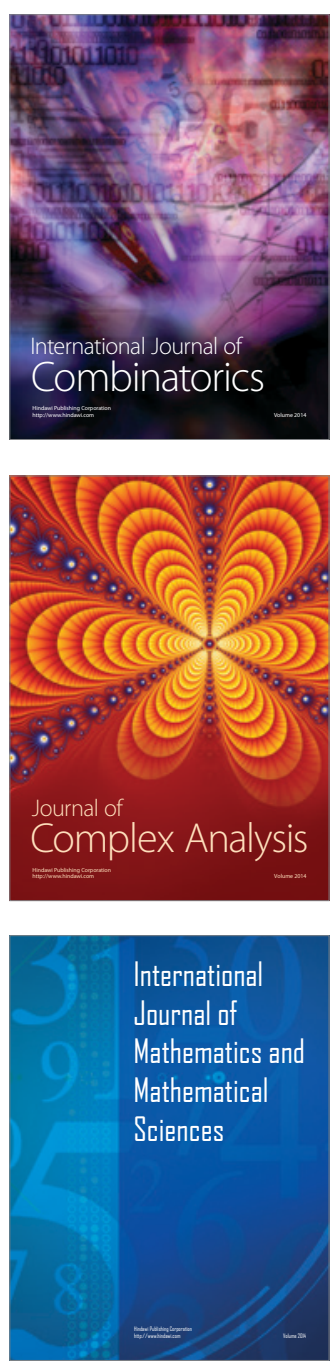
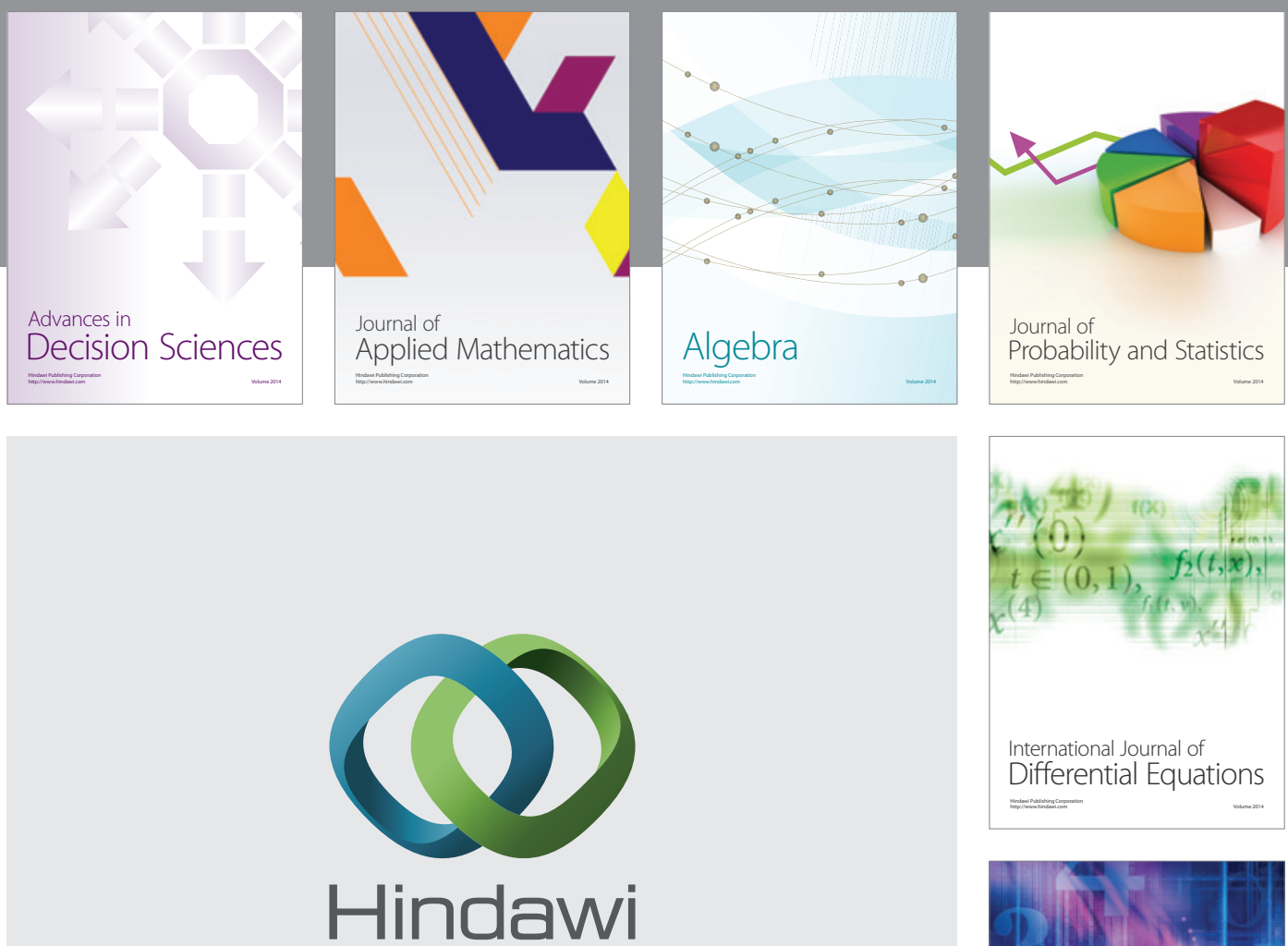

Submit your manuscripts at http://www.hindawi.com
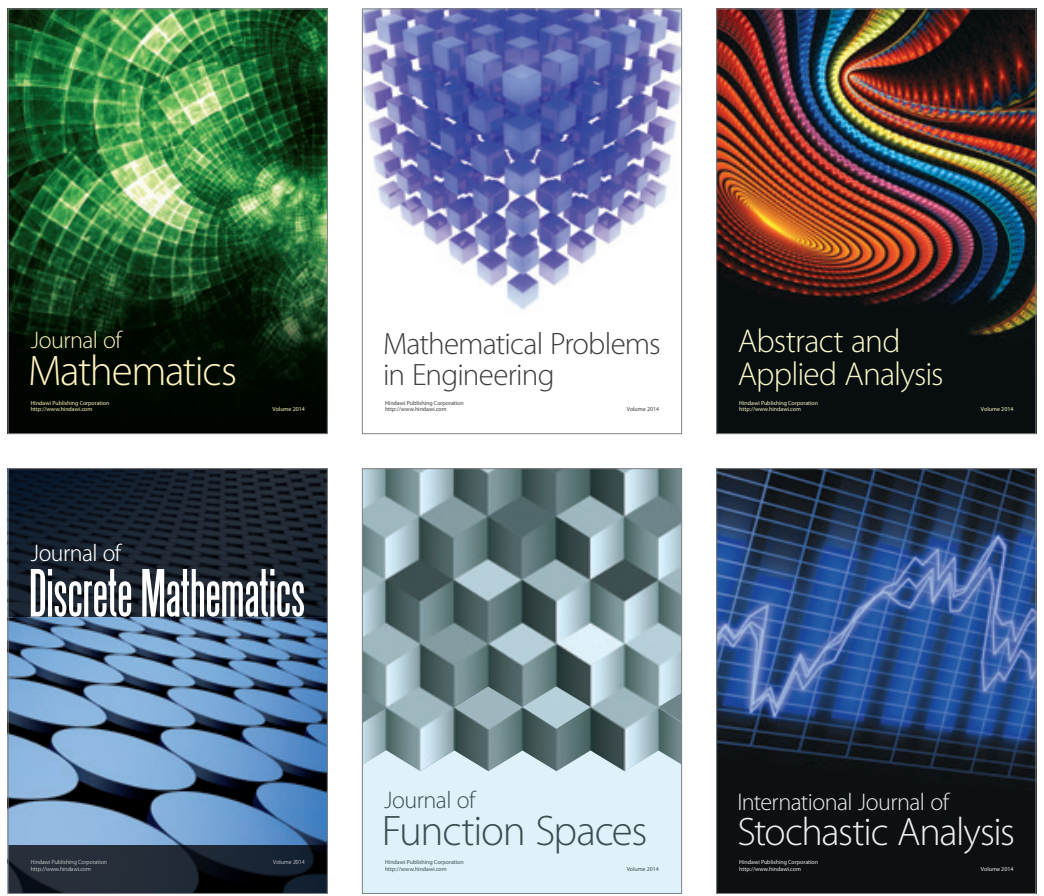

Journal of

Function Spaces

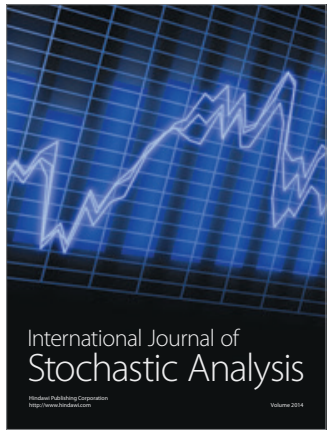

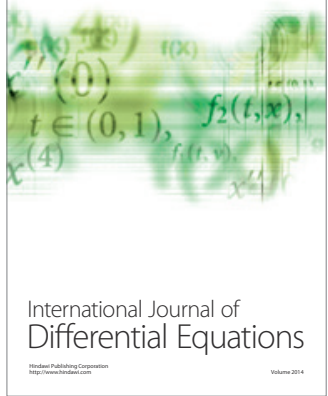
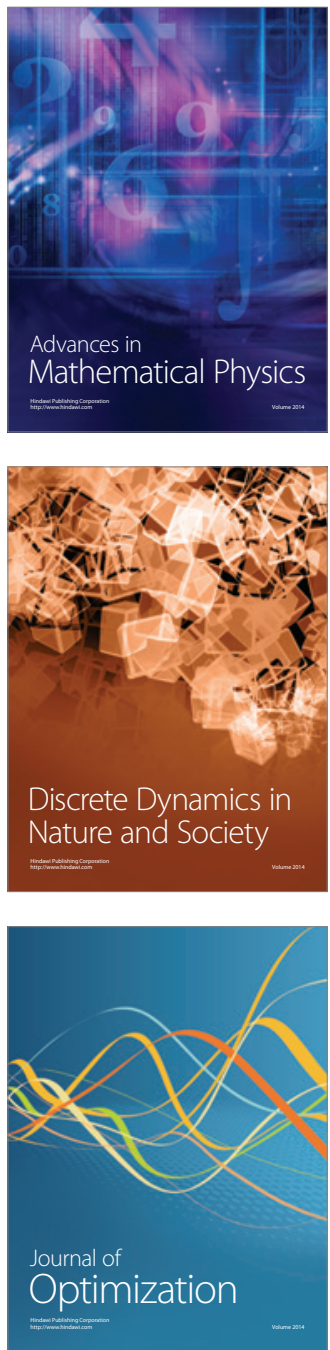